\title{
Balkanologie
}

Balkanologie Revue d'études pluridisciplinaires

Vol. X, $n^{\circ} 1-2 \mid 2008$

Volume $X$ Numéro 1-2

\section{Un réseau austro-hongrois à Zagreb à la fin du XIX siècle : August Šenoa et la revue littéraire Vienac}

Ellen Elias-Bursać

Traducteur : Jamila Chauvin

\section{OpenEdition}

Journals

Édition électronique

URL : http://journals.openedition.org/balkanologie/419

DOI : $10.4000 /$ balkanologie.419

ISSN : 1965-0582

Éditeur

Association française d'études sur les Balkans (Afebalk)

Référence électronique

Ellen Elias-Bursać, « Un réseau austro-hongrois à Zagreb à la fin du XIXe siècle : August Šenoa et la revue littéraire Vienac », Balkanologie [En ligne], Vol. X, n 1-2 | 2008, mis en ligne le 02 juin 2008, consulté le 17 décembre 2020. URL : http://journals.openedition.org/balkanologie/419 ; DOI : https:// doi.org/10.4000/balkanologie.419

Ce document a été généré automatiquement le 17 décembre 2020.

(c) Tous droits réservés 


\title{
Un réseau austro-hongrois à Zagreb à la fin du XIX ${ }^{\mathrm{e}}$ siècle : August Šenoa et la revue littéraire Vienac
}

\author{
Ellen Elias-Bursać
}

Traduction : Jamila Chauvin

1 C'est autour d'August Šenoa (1838-1881), premier romancier croate moderne et rédacteur en chef de la revue littéraire Vienac, que s'est définie la culture croate dans la seconde moitié du XIX ${ }^{e}$ siècle. Son travail au sein de la revue lui a permis de faire émerger une langue littéraire, de rassembler une génération de jeunes écrivains qui ont fait basculer la littérature croate dans la modernité, et de poser les fondations d'une identité nationale croate et d'un sentiment d'appartenance à une communauté culturelle. La langue croate s'était plus ou moins codifiée dans la première moitié du siècle, mais il restait à composer les romans qui pousseraient le grand public à lire et à écrire dans une autre langue que l'allemand; la grande réussite de Šenoa, comme auteur et comme animateur de revue, ce fut précisément de créer un lectorat.

2 Grâce à ses liens avec des écrivains de toute l'Autriche-Hongrie, Šenoa bâtit un réseau culturel qui contribua à définir pour les Croates un contexte géographique plus vaste : d'abord les territoires voisins - la Slovénie, la Bosnie-Herzégovine, la Serbie, le Monténégro -, puis les territoires polonais et tchèques au sein de l'Autriche-Hongrie, et enfin la France, l'Angleterre, la Russie, le Danemark, l'Ukraine, l'Italie, la Bulgarie, l'Albanie, la Prusse et d'autres pays encore.

Bien que né à Zagreb, donc en Croatie, August Šenoa a été décrit par l'un de ses biographes comme le "fils dénaturé d'un père tchèque germanisé et d'une mère slovaque "hongrianisée" "1. Les archives familiales établissent que ses ancêtres, originaires de Čáslav en Bohême, avaient vécu à Vienne puis à Budapest avant de s'établir en Croatie. Leur nom de famille s'est tour à tour orthographié Scheynoch, Schönau, Schöynoh, Schenoh, Schönoa. Ils avaient encore des parents à Budapest, et une tante cantatrice à Bucarest. 
4 Avant la naissance de Šenoa, son père était venu à Zagreb travailler comme pâtissier de l'évêque de la ville. À la maison, la famille parlait hongrois et allemand. Le jeune Šenoa parlait aussi allemand à l'école, pendant ses études primaires à Zagreb, comme tous les enfants de la période néo-absolutiste (les années 1850), durant laquelle l'usage de la langue croate était interdit à l'école comme dans toute la vie publique. Après une première année d'études secondaires à Pécs, en Hongrie, il suivit les cours du lycée de Zagreb. C'est alors que la fin du néo-absolutisme redonna une existence officielle à la langue croate. Dans ses souvenirs, il décrit ce moment comme un tournant décisif de sa vie, le début d'une adhésion passionnée à la culture croate.

5 À partir de 1858, il partagea son temps entre Prague et Vienne, où il étudia d'abord la médecine puis le droit, grâce à des bourses d'études que lui avait octroyées l'évêque, Mgr Strossmayer. C'est au cours de ces études qu'il se lia d'amitié avec des écrivains et des critiques de Prague, de Vienne et de Cracovie, tissant ce réseau intellectuel qui allait lui être si précieux en tant que collaborateur puis rédacteur en chef de Vienac. À Prague, il écrivit, pour les journaux Náródni listy, Hlas, Osvěta, Zlatá Praha, des articles en langue tchèque consacrés à l'actualité de la Croatie, du Monténégro, de la Serbie et de la Slovénie, tandis qu'à Vienne il publiait en allemand dans les Slavische Blätter et la österreichische Revue, ce qui lui permit d'élargir sa palette en rédigeant des essais, des notices biographiques, des nécrologies, et en consacrant des articles à la vie culturelle et aux écrivains tchèques et polonais; il y assuma en outre des responsabilités éditoriales. Pendant ce séjour à l'étranger, il adressa aussi des articles en langue croate à deux revues de Zagreb, Pozor et Glasonoša. Son essai « Naša književnost » (« Notre littérature»), paru dans Glasonoša, décrivait le type de littérature qu'il espérait promouvoir en Croatie :

J'ai déjà eu l'occasion de déclarer que notre littérature ne façonne pas notre vie sociale autant qu'elle le devrait. Je suis convaincu que l'engagement social revêt une importance cruciale pour le progrès et l'évolution de notre peuple tout entier. Tant que notre paysannerie ne sera pas mieux éduquée, tant que l'esprit national ne sera pas solidement ancré dans chaque ville, dans chaque bureau, dans chaque école, et par-dessus tout dans chaque famille - le véritable fondement de la vie de la nation et de l'État-, il ne saurait être question d'une vie nationale vigoureuse et harmonieuse ! [...]

Le plus bel exemple à suivre nous est offert par la littérature polonaise.

Tous ses fondateurs, les Mickiewicz, Krasinski, Kraszewski, Slowacki, Zaleski, etc. écrivaient, en poésie comme en prose, dans la perspective du progrès social ; est-il besoin de souligner l'effet bénéfique que ces grands hommes ont eu sur l'esprit polonais ??

6 De même, dans un article adressé à Pozor, il donnait en exemple les Tchèques et la création réussie d'un théâtre en langue tchèque à Prague :

Je suis particulièrement ravi d'aborder cette question et d'ouvrir le débat sur nos institutions théâtrales en fournissant un exemple tout à fait actuel: le théâtre tchèque, que j'ai vu mûrir de mes propres yeux. Ma première expérience, qui remonte à 1859, m'avait laissé une impression désastreuse : leur théâtre était aussi pitoyable que le nôtre, voire légèrement pire. Les acteurs étaient pour l'essentiel des débutants, leur jeu inexistant, et le répertoire d'une faiblesse accablante. Mais si vous aviez vu le chemin parcouru en quelques années, si vous aviez vu les Tchèques représenter, pendant une semaine entière, les plus grandes tragédies et comédies de Shakespeare, et glorifier ainsi sa mémoire! Le plus néophyte des amateurs d'art en aurait été transporté de joie. C'est ainsi qu'aujourd'hui, messieurs, la scène tchèque de Prague, qu'il s'agisse de théâtre ou d'opéra, est bien meilleure que la scène allemande. [...] 
Or, les Tchèques n'ont pas accompli ce prodige sous un quelconque gouvernement national; non, mes amis, ils l'ont accompli sous la tutelle de Schmerling, qui les méprise tant ; ils l'ont accompli sous un gouvernement à majorité allemand.

Qu'attendons-nous donc ?3

7 Šenoa revint à Zagreb en 1866, sans diplôme de droit, mais avec une vaste expérience de l'édition, et une vision très claire de ce que réclamaient la culture et la littérature croates. Lorsque la revue Vienac fut fondée en 1869 par l'Académie des sciences et des arts et la Matica Hrvatska (association culturelle et maison d'édition), il en devint l'un des collaborateurs les plus actifs, y publiant des critiques dramatiques, des nouvelles et des récits, des articles, et enfin ses cinq romans historiques sous forme de feuilletons ${ }^{4}$, à commencer par Zlatarovo zlato (L'Or de l'orfèvre), qui parut dans Vienac en 1871. Avant même de participer à la revue, il était le plus populaire des auteurs de langue croate.

En 1874, il fut nommé rédacteur en chef, poste qu'il conserva jusqu'à sa mort en 1881. Durant cette période, la revue comptait entre 1200 et 2000 abonnés, ce qui en fait le périodique de langue croate le plus lu de la seconde moitié du dix-neuvième siècle. Elle continua de paraître jusqu'en 1903, et plusieurs revues en ont successivement repris le nom ; l'incarnation actuelle de Vienac existe depuis 1993.

9 La revue privilégiait les textes traduits : nouvelles, poèmes, essais, romans-feuilletons, articles consacrés aux sciences, à la santé, à l'archéologie, à l'histoire et aux questions sociales. Bien souvent, à vrai dire, le nombre des traductions publiées dans un numéro excédait celui des textes écrits directement en croate. C'est dans cet esprit, et en faisant appel au cercle de ses connaissances, que Šenoa fit collaborer à Vienac des écrivains et traducteurs tchèques et polonais, dont la contribution conféra à la revue une ampleur de vues impressionnante. Régulièrement, elle publiait en traduction des œuvres d'auteurs français, tchèques, polonais, russes, allemands, bulgares et italiens, ou consacrait des articles à la culture et à la littérature de leurs pays; sans oublier des articles occasionnels portant sur les cultures albanaise, ukrainienne, roumaine, espagnole, slovaque, danoise, anglo-américaine et gréco-latine, et même un poème traditionnel péruvien.

10 Même si les traducteurs travaillaient souvent à partir des traductions allemandes existantes plutôt que des textes originaux, la grande victoire de Vienac fut d'élargir considérablement l'horizon culturel du lectorat croate, à une époque où la langue allemande exerçait une domination écrasante sur la vie culturelle et politique de Zagreb. Si, dans les années 1830 et 1840, Ljudevit Gaj avait encouragé la diffusion de journaux et de revues littéraires en langue croate parmi un public d'abonnés restreint mais fidèle, l'avènement du néo-absolutisme mit un terme à toute publication en langue croate. À l'époque de Šenoa, la plupart des quotidiens et périodiques étaient en langue allemande, tels l'Agramer Zeitung, l'Agramer Tagblatt, l'Agramer Wochenblatt. De fait, le rejet de toute influence autrichienne était si radical à Vienac que presque tous les auteurs germanophones publiés par la revue ${ }^{5}$ étaient originaires d'Allemagne, et non d'Autriche; et pendant toute la période où Šenoa y collabora, un seul écrivain hongrois figura au sommaire ${ }^{6}$. Si les écrivains autrichiens parvenaient à se faire publier dans la presse germanophone, il n'existait pas de presse en langue hongroise, si bien que la culture hongroise était complètement occultée à Zagreb. Ce fait est d'autant plus frappant que le hongrois était la langue maternelle de Šenoa, qui sans nul doute avait lu les œuvres des auteurs hongrois les plus marquants, et singulièrement les romans historiques de Mór Jókai, qu'il avait dû découvrir pendant son année de collège à Pécs. On attribue généralement le vif intérêt manifesté par Vienac pour la culture et la 
littérature françaises ${ }^{7}$ à une volonté de substituer l'influence de Paris à celle de Vienne ${ }^{8}$.

11 Le périodique tchèque Zlatá Praha,pour lequel Šenoa avait travaillé pendant ses années d'études, offrait un bon exemple de revue déterminée à redéfinir son contexte culturel et géographique en incluant des textes provenant (ou consacrés à) d'autres pays slaves, et en excluant tout élément germanique :

[Zlatá Praha] a redessiné la carte géographique en traitant la Bohême et la Moravie qui étaient à l'époque deux entités administratives séparées au sein de l'empire des Habsbourg - comme un espace national uni, dont elle soulignait plus largement la dimension slave dans sa rubrique "Le Monde slave" et dans ses nombreux dossiers consacrés à la Russie, à la Serbie, à la Bulgarie et autres territoires slaves; elle a donné un visage et un corps à la nation tchèque par ses articles nécrologiques et commémoratifs, ignorant résolument le Prague allemand et toute sa production. ${ }^{9}$

Dans le même esprit, chaque numéro de Vienac offrait à ses lecteurs des articles, des illustrations et des récits de voyage consacrés aux territoires voisins de la CroatieSlavonie: la Slovénie, la Dalmatie, la Serbie, le Monténégro, la Bosnie-Herzégovine. Compte tenu de la violente controverse qui fit rage entre 1849 et 1867 au sein de l'empire des Habsbourg, et singulièrement dans sa partie balkanique, quant au sort à réserver à ces territoires (fallait-il les diviser ou les réunir?), toute référence géographique ou territoriale - y compris dans les lithographies apparemment innocentes publiées par Vienac et représentant des scènes urbaines ou bucoliques dans des régions telles que la Dalmatie, la Serbie ou la Bosnie - était lourde de sens et forcément polémique. La géographie était un enjeu brûlant et crucial.

De 1700, date à laquelle la Croatie et la Slavonie avaient été réunies en une seule région par le régime de Budapest, à la révolution de 1848, ces deux territoires demeurèrent soumis à la Hongrie à l'intérieur de l'empire des Habsbourg. Lorsque, en 1848, pour obtenir la parité au sein de la monarchie, la Hongrie affronta l'Autriche, celle-ci, avec l'aide de troupes croates, remporta la victoire et annexa la Croatie-Slavonie aux terres de la Couronne autrichienne. Autrement dit, pendant une vingtaine d'années, la Croatie-Slavonie comme la Dalmatie furent placées sous administration directe de l'Autriche. Au début des années 1860, la communauté intellectuelle des territoires croates se déchira sur cette question : la plus grande menace pour le progrès culturel et politique croate résidait-elle dans une alliance étroite avec l'Autriche ou avec la Hongrie? Dans les faits, ce dilemme fut résolu par des facteurs extérieurs. En 1867, incapables d'assumer le coût de leurs campagnes militaires en Italie et en Prusse, les Autrichiens négocièrent avec les Hongrois un compromis aboutissant à un partage de l'autorité (l'Ausgleich ou Nagodba), qui devait permettre aux Habsbourg d'augmenter leurs ressources financières et leurs effectifs militaires.

Les treize années que passa Šenoa à Vienac virent se produire des bouleversements territoriaux considérables.La Croatie-Slavonie fut reléguée en Transleithanie (terres de la Couronne hongroise) par le Compromis de 1867, tandis que la Dalmatie demeurait en Cisleithanie (terres de la Couronne autrichienne). En 1870, la ville de Rijeka, alias Fiume, se vit conférer le statut spécial de "Corpus separatum» de la Couronne hongroise. En 1878, la Bosnie-Herzégovine, occupée par l'Autriche-Hongrie, fut considérée comme territoire impérial relevant de la double monarchie, administrativement distinct de la Dalmatie comme de la Croatie-Slavonie. Enfin, en 1881, la zone sous administration militaire retourna sous contrôle administratif croate. 

en langue croate), Prose ${ }^{11}$ (romans publiés en feuilletons et nouvelles, traduits ou écrits en croate), Articles éducatifs, Théâtre, Littérature, Biographies, Divers. Les «articles éducatifs » abordent une grande variété de sujets visant à l'instruction du lecteur et, dès la première année, manifestent une vaste curiosité géographique. On y trouve tour à tour des textes sur le rituel serbe de la slava, les écoles primaires en Prusse, les cafés et les journaux anglais à la fin du dix-septième siècle, des scènes de la vie bosniaque, la musique folklorique des Yougoslaves, les ballades slovènes, l'accès des Américaines à l'université. Sans oublier, dès la première année, des articles sur le rôle des femmes dans la société et sur l'éducation : les femmes et les progrès de la science, les droits et l'éducation artistique des femmes, vocation et patriotisme chez les femmes, ainsi que des suggestions concernant l'éducation des enfants, l'alphabétisation et l'initiation à l'art. Enfin, on y trouve des articles consacrés aux sciences, à l'agriculture, à l'hygiène ; parmi les sujets traités : l'astronomie, le sommeil, le tabac, les fruits, l'œil humain, la géologie, le vin, la combustion, les soins de la peau et du cheveu, les processus mentaux. d'histoire et de sciences sociales. Il créa une rubrique intitulée "Histoire, Lieux, Voyages, Peuples ${ }^{12}$, composée d'articles, d'illustrations et de commentaires audacieux sur des régions souvent chargées de symboles politiques, telles que, dans le volume de 1876 : Hvar (Dalmatie), Knin (zone sous administration militaire), Pula (Istrie), Solin (Dalmatie), Kiseg ${ }^{13}$, Solun (alias Salonique), Boka (Monténégro), Sarajevo, Belgrade, Sinj (Dalmatie), Skadar (Albanie), Dubrovnik (Dalmatie), Zadar (Dalmatie), Kiev, le Monténégro, Vučji dol ${ }^{14}$, Brač (Dalmatie), Niš (Serbie), la Bulgarie ou encore Imotski (Dalmatie).

Cette mise en perspective géographique s'enrichit d'échanges complexes entre les divers collaborateurs de la revue. Si par exemple Vienac offrit à ses lecteurs toute une série de traductions d'œuvres bulgares et d'articles consacrés à la Bulgarie, c'est uniquement parce que l'intérêt pour ce pays avait été ravivé par les textes d'un érudit tchèque, Konstantin Jiriček, qui y avait vécu plusieurs années et avait rendu compte de son séjour pour Vienac en 1877. Pour que les lecteurs croates s'intéressent aux territoires bulgares, il fallait d'abord qu'un universitaire tchèque leur prouve qu'ils étaient intéressants. De même, il fallut un écrivain russe pour aborder l'Albanie dans la revue : Pembe, le romande Konstantin Leontiev situé dans ce pays, parut en feuilleton dans Vienac en 1874, traduit du russe.

Les articles consacrés au Monténégro par Siegfried Kapper constituent un autre cas exemplaire. Kapper était un médecin juif tchèque auquel Šenoa s'intéressait depuis

Balkanologie, Vol. X, $n^{\circ} 1-2$ | 2008 
longtemps ${ }^{15}$. Fasciné par Vuk Karadžić et son projet de recueillir toute une tradition littéraire orale, Kapper s'était rendu dans les années 1840 à Karlovac, où il avait rencontré plusieurs représentants de l'illyrisme. Il consacra ensuite plusieurs récits de voyage - écrits en allemand et publiés à Leipzig - aux mœurs et à la culture des Balkans, et traduisit en allemand des récits appartenant à la tradition orale, recueillis par lui-même ou par Karadžić. Dans son avant-propos à une série d'articles de Kapper («Les Écoles du Monténégro » et « Femmes du Monténégro »), Šenoa remarque :

Le professeur Kapper, célèbre auteur tchèque, nous a fait parvenir toute une série d'articles fort intéressants consacrés au Monténégro, déjà soumis à la Deutsche Revue, et qui seront bientôt réunis en volume. Ces croquis extrêmement pittoresques, œuvre d'un grand écrivain, feront assurément les délices du lecteur de Vienac. » (1877, p. 566)

L'article de Kapper sur les femmes du Monténégro s'ouvre par une description extrêmement sévère du sort qui leur est réservé :

Plus un peuple est instruit, plus il respecte les femmes; plus il est primitif, plus elles sont opprimées.

Dans tout l'Orient, la femme n'est que l'esclave de son mari ; il en va de même au Monténégro. Le Monténégrin a honte de travailler, car c'est un « héros » qui porte une arme, fume sa pipe et ne fait rien. Toutes les tâches domestiques, tous les travaux des champs, ce sont les femmes qui les effectuent. Au marché de Kotor, il est fréquent de voir une femme porter sur ses épaules du bois ou autre fardeau, tandis qu'à ses côtés son mari chantonne nonchalamment. (1877, p. 807)

L'avant-propos laisse entendre que les lecteurs de Vienac risquaient de constater une différence de vision entre Kapper et les auteurs locaux. Peut-être la franchise du premier, et son refus du sentimentalisme, pouvaient-ils selon Šenoa dérouter le lecteur, ou du moins l'intriguer en lui présentant un sujet familier du point de vue d'un étranger.

Sous l'impulsion de Šenoa, Vienac consacra de nombreux textes aux droits des femmes, notamment au droit de vote, ainsi qu'aux femmes écrivains. À l'époque, le féminisme allait de pair avec la revendication nationaliste, comme le prouve l'exemple de Prague, où le "premier magazine féminin tchèque, Ženské listy, fut lancé par la féministe (et ardente patriote) Eliška Krásnohorská en $1875 »^{16}$.

La question de la place de la femme dans la société fut abordée dans des textes traduits d'auteurs étrangers avant d'être reprise par les écrivains locaux. Dès le deuxième numéro de la revue, le débat fut inauguré par un article de l'auteur britannique Henry Thomas Buckle, «L'Influence des femmes sur les progrès de la science ». L'année 1871 vit paraitre plusieurs articles sur le statut légal et social de la femme et l'émancipation féminine, ainsi qu'une étude biographique sur Madame de Staël et George Sand. Puis, en 1873, la revue publia un essai d'un auteur tchèque, $M$. Krajnik, sur les femmes écrivains slaves, auquel le traducteur croate joignit un appendice portant sur leurs homologues croates, serbes et slovènes.

24 L'efficacité d'une tel didactisme pour les lecteurs comme pour les collaborateurs de Vienac est parfaitement illustrée par le cas de Heinrich Sterneck ${ }^{17}$, l'un des rares auteurs autrichiens germanophones publiés par la revue. En 1878,il consacra un récit de voyage aux pierres levées de Bosnie, connues sous le nom de stećaks. Son article débutait ainsi :

Lors de mes nombreux voyages en Herzégovine et dans les régions voisines de Bosnie, j'ai découvert, à proximité de ruines romaines, d'étonnantes pierres tombales qui m'ont paru remarquablement intéressantes. 
À ma connaissance, jamais ces pierres n'ont été examinées par des experts ni même décrites nulle part, et je ne saurais manquer l'occasion d'attirer sur elles l'attention $\mathrm{du}$ public; car elles nous révéleront bien des secrets de l'histoire et de la géographie ; je suis d'avis qu'elles ont été édifiées à l'époque où ce territoire était encore cultivé, et donc apte à nourrir une population vaste et active. Peut-être ces monuments contribueront-ils à nous éclairer sur les grands bouleversements qu'ont subis ces contrées. (1878, pp. 549-550) en polonais et en hongrois ${ }^{18}$, cinq ans après sa parution en langue croate, la traduction tchèque à laquelle il fait allusion est l'œuvre de Marija Fabković, publiée à Prague en 1879 sous le titre Zlatnikův zlatoušek, tandis que la première traduction allemande, Das Goldkind, due à R. Podermanski, parut d'abord en feuilleton dans l'Agramer Zeitung en 1874, puis en volume chez Carl Albrecht, une maison d'édition germanophone de Zagreb. On ne trouve pas trace d'une traduction hongroise, malgré les affirmations de Šenoa. Peut-être traduisit-il lui-même son roman sans trouver d'éditeur. La traduction polonaise effectuée par Bronisław Grabowski parut à Varsovie en 1880 sous le titre Złota Dzieweczka. Grabowski ${ }^{19}$ militait à Cracovie pour la cause des Slaves du Sud, comme l'indique cet extrait d'un article de Vienac:

Les Polonais évaluent notre littérature. Depuis quelque temps, l'activité littéraire croate suscite de l'intérêt chez d'autres peuples, singulièrement les Polonais. G. Bronisłav Grabowski, professeur de lycée à Czestochowa, et Mme Pavlina Konarzewska, de Krževcih, ont joué un rôle déterminant pour faire connaître ces œuvres. M. Grabowski, lui-même poète d'expression polonaise, se consacre à l'étude de la 
littérature yougoslave et plus particulièrement croate, dont il a déjà traduit de nombreux textes en polonais, et promeut les ouvrages croates dans les revues de son pays. L'an dernier, il a traduit la nouvelle de Šenoa « Un œillet cueilli sur la tombe du poète ", parue dans la grande revue de Varsovie Tygodnik illustrowani; cette année, il a publié un panorama détaillé de la littérature croate actuelle dans les volumes 101 et 102 de Njive, autre revue varsovienne. Nous exprimons notre gratitude la plus chaleureuse à ce merveilleux auteur polonais, qui s'intéresse tant à notre littérature et qui la fait connaître à ses compatriotes. $(1879$, p. 210) de Cracovie. Lorsque en 1881 Zagreb fut frappée par un violent tremblement de terre, c'est Grabowski qui mit sur pied la publication d'un recueil vendu au bénéfice des victimes. Ce recueil est ainsi décrit dans Vienac:

Un ami de Cracovie nous a fait parvenir un superbe recueil en polonais, récemment publié par le «Cercle artistique et littéraire » de sa ville au profit des habitants de Zagreb victimes du tremblement de terre. Pour la deuxième fois en très peu de temps, l'intelligentsia polonaise exprime ainsi la sympathie de tout un peuple pour ses frères croates souffrants. Ce recueil, «Krakow - Zagrzebiowi ", comporte des poèmes, des textes en prose, des croquis et des compositions d'environ quatrevingts écrivains et artistes polonais, auxquels se sont joints deux Français : Jules Mien, connu par la «Revue Slave» comme un remarquable traducteur des œuvres de Słowacki, et le célèbre écrivain voyageur Victor Tissot, qui ont chacun offert un poème. $(1881, \text { p. } 89)^{20}$

Šenoa mourut des suites d'une crise de fièvre rhumatismale, à l'âge de quarante-trois ans, en 1881, au lendemain du tremblement de terre; mais il avait eu le temps de voir son labeur porter ses fruits. Si son projet initial visait simplement à définir une communauté culturelle pour les territoires croates en publiant des traductions de poètes et de romanciers polonais, il avait fini par forger un lien assez solide pour que, en retour, les Polonais expriment leur compassion et leur soutien non seulement à la poignée d'intellectuels et d'écrivains qui collaboraient à Vienac, mais à tous les habitants de Zagreb. La communauté utopique était devenue réalité.

\section{NOTES}

1. Jelčić (Dubravko), Šenoa, Globus, 1984, p. 50.

2. Glasonoša, 1865 ; Djela Augusta Šenoe, Globus, 1980, pp 8-9.

3. Pozor, 1866 ; Djela Augusta Šenoe, Globus, 1980, p. 52.

4. Zlatarovo zlato (L'Or de l'orfèvre), 1871 ; Čuvaj se senjske ruke (Prends garde à la main de Senj), 1875 ; Diogenes (Diogène), 1878; Seljačka buna (La Révolte des paysans), 1878; Kletva (La Malédiction), publication posthume.

5. Notamment Uhland, Goethe, Schiller et Heine.

6. En l'occurrence Petöfy, en 1875. Petöfy avait en réalité des origines serbes et s'appelait Aleksandar Petrović, jusqu'à ce qu'il change de nom par patriotisme pro-hongrois.

7. Voici la liste des auteurs français publiés par Šenoa dans Vienac: Jean Macé (1869), Deslys (1869), Voltaire (1870), Musset (1870, 1876, 1877), Madame de Staël (1871), George Sand (1871), Lamartine (1873), Lemoyne (1873), Chateaubriand (1874), Hugo (1874, 1875, 1878), Edmond About 
(1874), Mérimée (1875), Molière (1875), Racine (1875), Goncourt (1876), Jules Verne (1877), Daudet (1879), Zola (1881).

8. Šimundža (Drago), Francuska književnost u "Viencu”, Književni krug, Split, 1993, citant Nevenka Košutić-Brozović.

9. Sayer (Derek), "The Language of Nationality and the Nationality of Language: Prague 1780-1920 ", Past and Present, n 153 (Nov. 1996), p. 199.

10. Schiller, Byron, Goethe, Uhland, Krasinski, Geibel, Lermontov, Leopardi et Anacréon.

11. Kosinski, Tourgueniev, Jean Macé, Hálek, Gogol.

12. À l'époque, le sommaire était le suivant: Nouvelles; Poèmes; Biographies et Nécrologies ; Histoire, Descriptions de lieux, Récits de voyage, Descriptions de peuples ; Sciences naturelles ; Littérature, Critique dramatique et artistique, Chroniques littéraires et mondaines; Art et Archéologie ; Illustrations ; Composition musicale.

13. Appelée Köszeg en hongrois, Kiseg fut assiégée au seizième siècle par l'armée ottomane de Soliman et victorieusement défendue, malgré leur infériorité numérique, par les troupes croates de Nikola Jurišić, qui reçut la ville en récompense.

14. Ville du Monténégro où, en 1867, une poignée de Monténégrins avait vaincu des troupes ottomanes supérieures en nombre.

15. Šenoa consacra un article à l'œuvre de Kapper pour les Slavische Blätter pendant ses années d'études à Vienne.

16. Sayer (Derek), p. 198.

17. Auteur de Geographische Verhältnisse, Communicationen und das Reisen in Bosnien, der Herzegovina, und Nord-Montenegro, Vienne, 1877.

18. «Voilà plusieurs années que je m'essaie à écrire des œuvres de fiction en langue croate. Le fruit de ces efforts a été publié de façon dispersée dans diverses revues croates, et certains textes demeurent inédits. J'ai donc décidé de les rassembler, de les corriger chaque fois que ce serait nécessaire, et d'en éliminer toute page ratée ou immature; ainsi amendées, mes œuvres paraîtront en une série de volumes. Jamais je ne m'y serais résolu si mes amis ne m'y avaient pas poussé, et si je n'avais pas constaté que les lecteurs croates apprécient mon travail ; et ce qui m'a encouragé dans ce dessein, c'est de voir certaines de mes œuvres traduites dans d'autres langues (ainsi L'Or de l'orfêvre a-t-il été traduit en allemand, en tchèque, en polonais et en hongrois, et Prijan Lovro en tchèque et en slovène). "

19. Jelčić (D.), p. 65.

20. Parmi les artistes ayant contribué à ce recueil figurent Jan Matejko, Wojciech et Zofia Kossak, Adam Asnyk, la poétesse Deotyma, Bronisław Grabowski, grand défenseur de la littérature croate, le célèbre Józef Kraszewski, Marian Gorzkowski, Aleksander Fredro, Michał Bałucki, Leon Kozłowski, Stanisław Smolka, Józef Szujski, Stanisław Koźmian, Wincenty Rapacki, Stefan Grudziński et Jan Zacharyasiewicz.

\section{RÉSUMÉS}

C'est autour d'August Šenoa (1838-1881), premier romancier croate moderne et rédacteur en chef de la revue littéraire Vienac, que s'est définie la culture croate dans la seconde moitié du XIX ${ }^{\mathrm{e}}$ siècle. Grâce à ses liens avec des écrivains de toute l'Autriche-Hongrie, Šenoa bâtit un réseau culturel qui contribua à définir pour les Croates un contexte géographique plus vaste : d'abord les 
territoires voisins - la Slovénie, la Bosnie-Herzégovine, la Serbie, le Monténégro -, puis les territoires polonais et tchèques au sein de l'Autriche-Hongrie, et enfin la France, l'Angleterre, la Russie, le Danemark, l'Ukraine, l'Italie, la Bulgarie, l'Albanie, la Prusse et d'autres pays encore.

INDEX

Mots-clés : Langue croate, Littérature croate

Index géographique : Autriche-Hongrie, Croatie, Zagreb 